\title{
Prevalence of phimosis and foreskin sliding abnormalities in male adolescents and their correlation with later onset of first sexual intercourse
}

\author{
Giuseppe La Pera $^{1}$, Francesco De Luca ${ }^{1,2}$, Attilio Guerani ${ }^{3}$, Alessandro Palmieri ${ }^{4}$, Giorgio Franco ${ }^{2}$ \\ ${ }^{1}$ Department of Urology Azienda Ospedaliera San Camillo Forlanini, Rome, Italy; \\ ${ }^{2}$ Department of Gynaecological and Urological Sciences, Sapienza University of Rome, Rome, Italy; \\ ${ }^{3}$ General Practitioner, Rome, Italy; \\ ${ }^{4}$ Department of Urology University of Naples, Naples, Italy
}

\begin{abstract}
Summary Introduction and objectives: The aim of the study is to evaluate the prevalence of andrological abnormalities, such as phimosis and foreskin sliding abnormalities among male adolescents, and if these might interfere with sexuality, leading to a later onset of sexual experiences.

Material and methods: Between April and May 2015 a prevention campaign in andrology was conducted in an area surrounding Rome, Ostia and the Ladispoli area, among 15-19 year-old students. The screening consisted of a frontal lesson with the students in order to explain and raise the awareness of the most common andrological abnormalities and diseases. Among the routine anamnestic questions, three additional questions were submitted to 18-year-old boys: "Have you ever had sexual intercourse?", "How old were you when you had your first sexual intercourse?" and "Have you consulted a health professional about your genitals?" Finally a detailed clinical examination was performed and the outcome sent to the family and to the General Practitioner (GP).

Results: A total of 552 high school students were evaluated. Out of them 131 (23.7\%) were at least 18 years old. Among these, $79(60.3 \%)$ said that they had already had full sexual intercourse. The phimosis and foreskin sliding abnormalities had a prevalence of $12.9 \%$ within the 18-year-old students, with a significant prevalence among those who hadn't had any sexual intercourse at all, $21.1 \%$ vs $7.5 \% p=0.023$. The age of the complete first sexual experience in the circumcised young men was the same as those without phimosis; $89 \%$ of the boys with phimosis hadn't had an andrological examination in the previous years.

Conclusions: Male adolescents with phimosis or preputial sliding abnormalities tend to have a late onset of sexual experiences compared to same aged boys without phimosis.

These data support the urgent need of an andrological consultation for all boys at the beginning of, and during, their adolescent period because genital abnormalities may interfere with sexuality. Finally, in order not to confuse effects with causes, we suggest matching a routine genital physical examination in all studies dealing with sexual psychological aspects of male adolescents.
\end{abstract}

KEY WORDS: Phimosis; Foreskin sliding abnormalities; Male adolescents; First sexual intercourse; Tight foreskin.

Submitted 21 September 2017; Accepted 8 October 2017

\section{INTRODUCTION}

Phimosis is defined as the inability of the prepuce (foreskin) to be retracted behind the glans penis in uncircumcised males. The prepuce is unretractable in almost $100 \%$ of infants and less than $7 \%$ of children approaching puberty. The prevalence decrease with the age (1). It may occurs mainly in childhood for congenital factors, while in adulthood it is often the result of chronic diseases of the penis or glans and/or recurrent infections that lead to foreskin and glans adhesions (2).

Little has been written about the presence of phimosis and foreskin sliding disorders during male adolescence (3), especially if this may affect approaching sex.

The aim of this study is to evaluate in a population of male adolescents the prevalence of phimosis and other foreskin sliding disorders and to assess whether these conditions could somehow affect a different approach to sex, such as a late onset of first complete sexual intercourse.

\section{MAteriAL AND Methods}

Between April and May 2015, the Italian Society of Andrology (SIA) and Italian Association for Sexual Right (AIDASS) conducted a prevention campaign in andrology. It was carried out in the municipality of Ladispoli and in the borough of Ostia, 30 kilometres away from Rome. All the boys between 15 and 19 years old and living in the municipality of Ladispoli were invited to take part in the survey (a total of 1180 boys), while in the borough of Ostia all the boys between 15 and 19 years old attending four high schools were invited. First of all we sent a letter to the family to explain the aim of the campaign and to obtain their written consent for a genital examination for adolescents under 18 years old. The screening was carried out in high schools and at general practitioner (GP) practice. It consisted of lessons with the students in order to explain and raise the awareness of the most common andrological diseases and how to prevent it, showing how to perform testicular self-examination for the prevention of testis cancer.

All students underwent to a genital examination by at least one urologist in which the definition of phimosis 
and foreskin sliding abnormalities had been defined according to these three situations:

1. the typical phimosis in which the foreskin cannot be pulled back and past the glans;

2. functional phimosis, in which the foreskin cannot be pulled back past the glans only during erection;

3. frenulum brevis is too short to allow complete foreskin retraction.

If one of the above conditions was present then the subject was considered to be affected by phimosis or foreskin sliding abnormalities. The chi-square test was used to analyze the difference in prevalence between those who had already had full sexual intercourse and those who had not. For all students older than 18 years, two additional questions were asked: "Have you ever had sexual intercourse and how old were you when you first had sexual intercourse?" and "Have you consulted a health professional about your genitals?" At the end, another correspondence was sent to the family and to the GP to let them know the final report.

The Local Ethical Committee was informed of the noninterventional and observational nature of the study.

The primary end point of the study was to evaluate the prevalence of phimosis and foreskin sliding abnormalities among male adolescents.

The secondary objectives were:

- to correlate genital abnormalities with late onset of sexual experiences;

- to teach self examination to all adolescents aged 15-19 years.

This latter aspect, and all results regarding other diseases such as varicocele and its prevalence in boys under 18 years old, will be analyzed in a separate paper.

\section{Results}

A total of 552 high school students were evaluated; 131 (23.7\%) were at least 18 years; among these, 79 (60.3\%) stated that they had already had full sexual intercourse. In more detail, 8 students had their first sexual experience at the age of 14,19 at the age of 15,18 at the age of 16 , 23 at the age of 17 and 11 at the age of 18 (Figure 1).

Phimosis and foreskin sliding abnormalities were found in

\section{Figure 1.}

Age at first sexual experience.

In column number and percentage distribution of students.

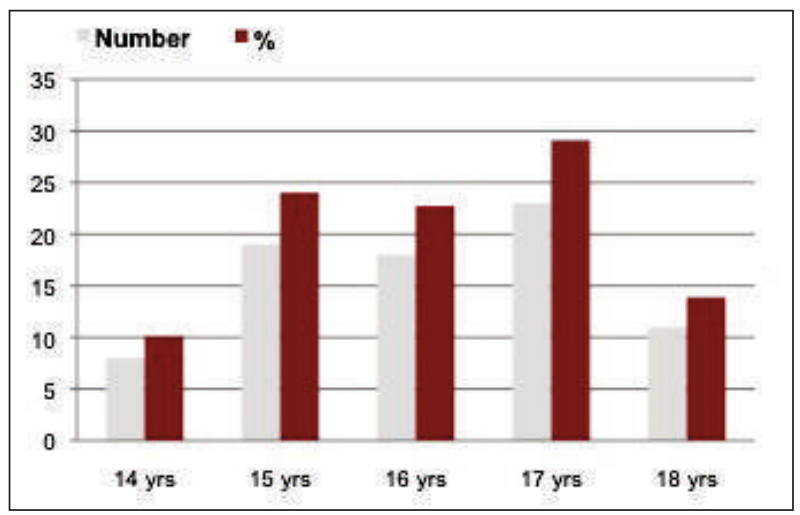

17 cases with a prevalence of $12.9 \%$ within the 18 -yearold students. There was a higher prevalence of phimosis among students who hadn't had any sexual intercourse at all: 11 subjects $(21.1 \%)$ versus $7(7.5 \%)$ students who had had complete sexual experiences (Figure 2).

Figure 2.

Number of subjects with or without phimosis and distribution according to their sexual experiences.

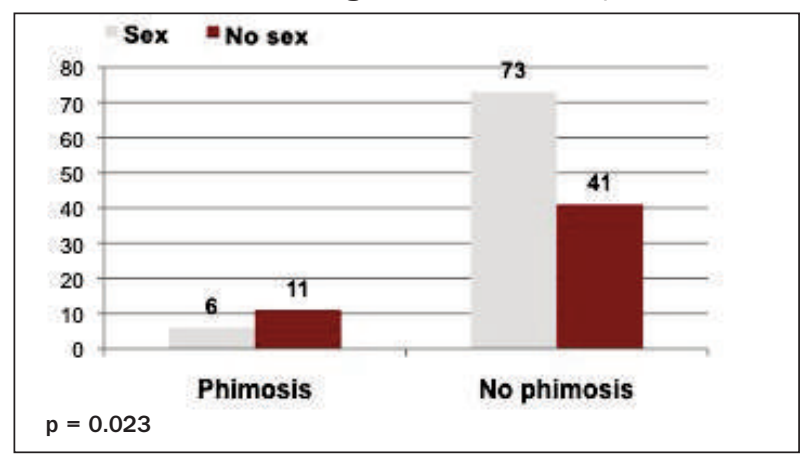

The chi-square test was 5.105 with $\mathrm{p}=0.023$.

The age of the first sexual experience of the circumcised students was the same as those without phimosis.

Furthermore, $89 \%$ of subjects with phimosis never had a previous genital examination.

\section{Discussion}

This study shows that the prevalence of phimosis and foreskin sliding disorders is significantly higher among those who have not had sexual intercourse (21.1\%) than among those who have already had sexual intercourse (7.5\%).

As shown in literature, the mean age of the first sexual experience is at 16 years (4). These observations show that these boys may face two problems, a biological one and a behavioural one. Biological because the degree of phimosis may worsen and lead to a urinary tract infection and because of the hypersensitivity of the glans that leads to premature ejaculation and discomfort or pain during masturbation or sexual intercourse. Also, phimosis and sexual disorders can interfere with sexuality, triggering behaviours that can lead to avoidance of sexual intercourse. This is shown by the observation that young men with phimosis tend to have less sexual intercourse than those who do not have phimosis.

This observation on adolescent behaviour is new compared to other similar studies (4) because, for the first time, it has been observed that the physical handicap could interfere with their approach to sex, such as delaying their first sexual intercourse, and in some cases could be the cause of psychological fragility. The delayed onset of sexual intercourse may be an adaptive behaviour of a physical disease and not the consequence of a primitive psychological fragility that arises subsequently. This adaptive behaviour has already been demonstrated in other sexual diseases, such as sexual dysfunction, which can trigger substance addiction $(5,6)$ and a similar mechanism can be the basis of the delayed onset of sexual intercourse.

Many psychological studies on adolescents' sexuality and behaviour are based only on questionnaires $(7,8)$, and 
therefore are not complete in our opinion. In this study we completed the questionnaires with a meticulous examination of the male genitals. Accordingly, we correlate the behavioural component with the medical one; this is something new, opening a wide window on the adolescents' behaviour. In our opinion, studies that investigate adolescent behaviour should be matched with extensive genital examination. Because, as shown in this study, some effects may be confused with the causes. This study underlines the importance of offering an andrological screening to all the family in order to prevent and treat andrological disease and promote male sexual health. This is also a way to promote and raise awareness of the importance among adolescents of andrological prevention, and to reduce the social embarrassment that late diagnosis can involve, as well as to explain to young men that there is a dedicated place to discuss sexual issues (9).

Another good point is to create a network between the General Practitioner and the specialist consultant.

A critical point is that those percentages may be underestimates. In fact this study is completely based on questionnaires, lessons and clinical examinations, mainly performed at school. We know really well that, even though public instruction is compulsory in Italy until the age of 16 , those adolescents who are at a disadvantage economically and socially may not be reached, as they will quit or have never been to school. These people are the ones who need more social and medical assistance. In our modest opinion, this highlights a relatively new figure of the uro-andrologist, being the counterpart of the gynaecologist. This person should play an important role in society and should be independent from the urologist. We all know that in Italy, in our National Health System (NHS) the priority is given to general urology (prostate, bladder and kidney diseases) ahead of the andrological conditions. In fact they are seen as minority patients, as it for was urology at the beginning when it was still included in general surgery and the urologist was seen as a minor surgeon. This should happen for andrology, and it should have its own independence, separate from urology, with its own unit and allocated budget $(9,10)$.

\section{Conclusions}

$21.1 \%$ of adolescents with phimosis and other foreskin sliding abnormalities haven't had any sexual experience at the age of 18 , compared to the same aged boys without phimosis. $89 \%$ of boys with phimosis did not receive any andrological consultation during adolescence.

The direct consequence of this observation is evidence of an urgent need of a routine andrological consultation in all boys at the beginning and during the adolescence period, as the genital pathologies are quite frequent.

Also, the data suggests the hypothesis that physical genital pathologies act as a cause of late onset of sexual experience and could be a trigger point of psychological fragility. Although we suggest matching physical andrological examinations in all studies dealing with sexual male adolescents' behaviour, more studies are needed to demonstrate that psychological fragility is the aftermath of andrological disease such as, but not limited to, phimosis and foreskin sliding disorders during adolescence.

\section{FINANCIAL SUPPORT}

The Administration of Regione Lazio and the Italian Society of Andrology partially supported this study.

\section{ACKNOWLEDGMENT}

We thank for their participation to the andrological campaign and for the andrological screening: Barrese $F$, Bitelli M., Boffini A., Bordacconi S., Buonprisco F., Chiriatti A., Ciletti M., Florio M., Fraschetti M., Fucito G., Gandini A., Iannotta L., Lauretti S., Marianantoni Z., Misuraca L., Rizzo G., Tassi B., Travaglia S., Tronino M., Sanpalmieri M., Santagata E., Santini E., Vaggi L.

We also thank the following General Practitioners from Ladispoli and Cerveteri: Alesiani MR., Aureli A., D'Amico MN., Fanini M., Faris R., Gentili G., Gionangeli Sebasti I., Paliotta C., Spaziani B., Valeri M.

Special thanks to the Administration of Ladispoli and the Mayor Paliotta C. and to Zaccari C. Head of primary care of ASL Roma F.

\section{REFERENCES}

1. Yang C, Liu X, Wei GH. Foreskin development in 10421 Chinese boys aged 0-18 years. World J Pediatr. 2009; 5:312-5.

2. Westwood M, Pinzon J. Adolescent male health. Paediatric Child Health 2008; 13:31-36.

3. Rizzotto A. Evidenziazione delle anomalie genital esterne alla visita di leva. Giornale Italiano di Andrologia. 1997; 3:107-111.

4. Vasilenko SA, Ram N, Lefkowitz ES. Body image and first sexual intercourse in late adolescence. J Adolesc. 2011; 34:327-35.

5. La Pera G, Franco Giannotti C, Taggi F, Macchia T. Prevalence of sexual disorders in those young males who later become drug abusers. J Sex Marital Ther. 2003; 29:149-56.

6. a Pera G, Carderi A, Marianantoni Z, et al. Sexual dysfunction prior to first drug use among former drug addicts and its possible causal meaning on drug addiction: preliminary results. J Sex Med. 2008; 5:164-72.

7. Carpenter LM. The ambiguity of "having sex": The subjective experience of virginity loss in the United States. J Sex Res. 2001; 38:127-139.

8. Trani F, Gnisci F, Nobile CG, Angelillo IF. Adolescents and sexually transmitted infections: knowledge and behaviour in Italy J Paediatr Child Health. 2005; 41:260-4.

9. Mondaini N, Silvani M, Zenico T, et al. Genital diseases awareness in young male students: Is information necessary to protect them? Arch Ital Urol Androl. 2013; 85:14-9.

10. Foresta C, Pizzol D. He had always wanted to ask an andrologist but had never done so. World J Clin Cases. 2014; 2:546-51.

\section{Correspondence}

Giuseppe La Pera, MD (Corresponding Author)

dr.giuseppelapera@gmail.com

Department of Urology Azienda Ospedaliera San Camillo Forlanini, Rome, Italy

Francesco De Luca, MD - francescodeluca10@gmail.com

Department of Gynaecological and Urological Sciences, Sapienza University of Rome, Rome, Italy

Attilio Guerani, MD - dr.attilioguerani@gmail

General Practitioner, Rome, Italy

Alessandro Palmieri, MD - alessandro.palmieri@unina.it Department of Urology University of Naples, Naples, Italy

Giorgio Franco, MD - giorgio.franco@libero.it

Department of Gynecological and Urological Sciences, Sapienza University of Rome, Rome, Italy 